\title{
A Cumulative Study on Differential Transform Method
}

\author{
Geeta Arora, Pratiksha* \\ Department of Mathematics \\ Lovely Professional University, Phagwara, Punjab, India \\ ${ }^{*}$ Corresponding author: text2pratiksha@ gmail.com
}

(Received May 19, 2018; Accepted August 6, 2018)

\begin{abstract}
Many real-world phenomena when modelled as a differential equation don't generally have exact solutions, so their numerical or analytic solutions are sought after. Differential transform method (DTM) is one of the analytical methods that gives the solution in the form of a power series. In this paper, a cumulative study is done on DTM and its evolution as an effective method to solve the gamut of differential equations.
\end{abstract}

Keywords- Differential transform method, Fractional differential equations, Fractional DTM.

\section{Introduction}

Whatever is happening around us, can be modelled as a mathematical expression, most commonly as a differential equation. The solution of that differential equations leads to better understanding of that phenomenon. If the equation can be solved by a comprehensive process using basic mathematical tools like calculus, trigonometry, geometry, algebra etc, it is called the analytic method; though it's a tough nut to crack, it's more reliable. On the other hand, a numerical method is more of an experimental nature. One may have to compromise on accuracy in numerical solutions. This work is to report and discuss the development in method of differential transform (DTM) which is commonly categorized as an analytical method (Arikoglu et al., 2007; Erturk et al., 2008; Al-Smadi et al., 2017) or termed as numerical method (Erturk et al., 2008; Momani et al., 2008) or semi-analytical / numerical method (Cetinkaya et al., 2011; Gokdogan et al., 2012) also. Like any other area of mathematics, DTM has grown over a period of time, longitudinally and latitudinally. It has been used to solve various types of differential equations including ordinary, partial and fractional differential equations. The intent of this study is to elaborate on the evolution of the method with a discussion on the recent trends and applications of this method.

This manuscript is prearranged in sections as follows: Section 2 introduces the differential transform for integer order derivatives for a function of one and two variables. Next section takes the idea further tor fractional order derivatives for the function of one and two variables. Section 4 presents some of the extensions done in DTM along with its usage by various authors. The last section summarizes the reported work in this manuscript with a discussion of future perspective.

\section{Method of Differential Transform}

The foundation of the DTM was conceived in 1986 by Zhou (Zhou, 1986). He solved initial value problems of linear and nonlinear nature arising in circuit analysis, using this transform. This method is similar to Taylor's series method and gives a polynomial kind of solution. This method does not involve the complexities of linearization and discretization. Moreover, it is free from initial guesswork too. 
International Journal of Mathematical, Engineering and Management Sciences

Vol. 4, No. 1, 170-181, 2019

https://dx.doi.org/10.33889/IJMEMS.2019.4.1-015

For understanding the concept of DTM, a differentiable function say $\phi(t)$ be represented by a series centred at $t_{i}$ in domain $D$ and $t_{i}$ be any value in the domain. The series expansion of function $\phi(t)$ due to Taylor is:

$\phi(t)=\sum_{r=0}^{\infty} \frac{\left(t-t_{i}\right)^{r}}{r !}\left[\frac{d^{r} \phi(t)}{d t^{r}}\right]_{t=t_{i}}, t \in D$.

At $t_{i}=0$ Taylor's series becomes Maclaurin's series, which leads to the definition of the differential transform.

For the $r$ th differential of a function $\phi(x)$, the differential transform is given by

$\Phi(r)=\frac{1}{r !}\left(\frac{d^{r} \phi(x)}{d x^{r}}\right)_{x=x_{0}}$.

and the inverse differential transform of $\Phi(r)$ is given by $\phi(x)=\sum_{r=0}^{\infty} \Phi(r)\left(x-x_{0}\right)^{r}$.

In actual problem solving, the inverse differential transform is taken as a finite sum for $n$ values and the sum of $(n+1)$ onwards terms is considered negligibly small.

Let us assume that the differential transforms of $\phi(x), \psi(x)$ and $\omega(x)$ are given by $\Phi(r), \Psi(r)$ and $\Omega(r)$ respectively. Differential transforms for the standard functions of one variable (Zhou, 1986) are reported in Table 1.

Table 1. Differential transforms

\begin{tabular}{|l|l|}
\hline \multicolumn{1}{|c|}{ Original Function } & \multicolumn{1}{c|}{ Transformed Function } \\
\hline$\phi(x)=\psi(x) \pm \omega(x)$ & $\Phi(r)=\Psi(r) \pm \Omega(r)$ \\
\hline$\phi(x)=c \psi(x)$ & $\Phi(r)=c \Psi(r), \quad c$ is a constant \\
\hline$\phi(x)=\psi(x) \omega(x)$ & $\Phi(r)=\sum_{i=0}^{r} \Psi(i) \Omega(r-i)$ \\
\hline$\phi(x)=\frac{d^{m} \psi(x)}{d x^{m}}$ & $\Phi(r)=\frac{(r+m) !}{r !} \Psi(r+m)$ \\
\hline$\phi(x)=x^{m}$ & $\Phi(r)=\delta(r-m), \quad \delta$ is Dirac delta function \\
\hline
\end{tabular}

Using this transform many ordinary differential equations (Erturk et al., 2007; Biazar et al., 2010) with their linear and non-linear structures (Mirzaee, 2011) have been successfully solved for the numerical solution. 
International Journal of Mathematical, Engineering and Management Sciences

Vol. 4, No. 1, 170-181, 2019

https://dx.doi.org/10.33889/IJMEMS.2019.4.1-015

\subsection{DTM in 2-Dimensions}

For a function with two independent variables, the DTM was developed by Chen et al., in the year 1999. The basic definition of differential transform for a given function $\omega(x, y)$ in two dimensions is given by

$\Omega(r, s)=\frac{1}{r ! s !}\left(\frac{\partial^{r+s} \omega(x, y)}{\partial x^{r} \partial y^{s}}\right)_{(0,0)}$.

The inverse differential transform $\omega(x, y)$ of $\Omega(r, s)$ is given by

$\omega(x, y)=\sum_{r=0}^{\infty} \sum_{s=0}^{\infty} \Omega(r, s) x^{r} y^{s}$.

Let us assume that $\Phi(r, s), \Psi(r, s)$ and $\Omega(r, s)$ are the differential transforms of $\phi(x, y), \psi(x, y)$ and $\omega(x, y)$ respectively. Table 2 contains the differential transforms (Ayaz, $2003)$ for the stated functions of two variables.

Table 2. Two dimensional differential transforms

\begin{tabular}{|l|l|}
\hline \multicolumn{1}{|c|}{ Original Function } & \multicolumn{1}{|c|}{ Transformed Function } \\
\hline$\phi(x, y)=\psi(x, y) \pm \omega(x, y)$ & $\Phi(r, s)=\Psi(r, s) \pm \Omega(r, s)$ \\
\hline$\phi(x, y)=c \psi(x, y)$ & $\Phi(r, s)=c \Psi(r, s), c$ is a constant \\
\hline$\phi(x, y)=\psi(x, y) \omega(x, y)$ & $\Phi(r, s)=\sum_{i=0}^{r} \sum_{j=0}^{s} \Psi(i, s-j) \Omega(r-i, j)$ \\
\hline$\phi(x, y)=\frac{\partial^{m+n} \psi(x, y)}{\partial x^{m} \partial y^{n}}$ & $\Phi(r, s)=\frac{(r+m) !(s+n) !}{r ! s !} \Psi(r+m, s+n)$ \\
\hline$\phi(x, y)=x^{m} y^{n}$ & $\Phi(r)=\delta(r-m) \delta(s-n), \delta_{\text {is Dirac delta function }}$ \\
\hline
\end{tabular}

\subsection{Reduced DTM}

In 2009, Turkish mathematicians (Keskin et al., 2009) had devised the method of reduced differential transform (RDTM) which as per the name reduced a variable and led to easier calculations. Consider a function $\phi(x, t)$ representable as a multiplication of a separable function in $x$ and $t$, i.e. $\phi(x, t)=\delta(x) \theta(t)$.

Using the characteristics of DTM mentioned in the previous section, the function $\phi(x, t)$ can be written as follows:

$\phi(x, t)=\sum_{i=0}^{\infty} \Delta(i) x^{i} \sum_{j=0}^{\infty} \Theta(j) t^{j}=\sum_{r=0}^{\infty} \Phi_{r}(x) t^{r}$

where $\Phi_{r}(x)$ is called spectrum function of $t$ dimension of $\phi(x, t)$. 
International Journal of Mathematical, Engineering and Management Sciences

Vol. 4, No. 1, 170-181, 2019

https://dx.doi.org/10.33889/IJMEMS.2019.4.1-015

Let $\phi(x, t)$ is an analytic function with respect to both independent variables in the considered domain, and then the transformed function (t-dimensional spectrum) $\Phi_{r}(x)$ is given as

$\Phi_{r}(x)=\frac{1}{r !}\left[\frac{\partial^{r} \phi(x, t)}{\partial x^{r}}\right]_{t=0}$.

The inverse differential transform function of $\Phi_{r}(x)$ is given by $\phi(x, t)=\sum_{r=0}^{\infty} \Phi_{r}(x) t^{r}$.

The rules for RDTM are mentioned in Table 3.

Table 3. Reduced differential transforms

\begin{tabular}{|l|l|}
\hline \multicolumn{1}{|c|}{ Original Function } & \multicolumn{1}{|c|}{ Transformed Function } \\
\hline$\phi(x, t)=\psi(x, t) \pm \omega(x, t)$ & $\Phi_{r}(x)=\Psi_{r}(x) \pm \Omega_{r}(x)$ \\
\hline$\phi(x, t)=c \psi(x, t)$ & $\Phi_{r}(x)=c \Psi_{r}(x), c$ is a constant \\
\hline$\phi(x, t)=\psi(x, t) \omega(x, t)$ & $\Phi_{r}(x)=\sum_{i=0}^{r} \Psi_{i}(x) \Omega_{r-i}(x)$ \\
\hline$\phi(x, t)=\frac{\partial^{m} \psi(x, t)}{\partial t^{m}}$ & $\Phi_{r}(x)=\frac{(r+m) !}{r !} \Psi_{r+m}(x)$ \\
\hline$\phi(x, t)=\frac{\partial \psi(x, t)}{\partial x}$ & $\Phi_{r}(x)=\frac{\partial}{\partial x} \Psi_{r}(x)$ \\
\hline$\phi(x, t)=x^{m} t^{n}$ & $\Phi_{r}(x)=x^{m} \delta(r-n), \delta$ is Dirac delta function \\
\hline
\end{tabular}

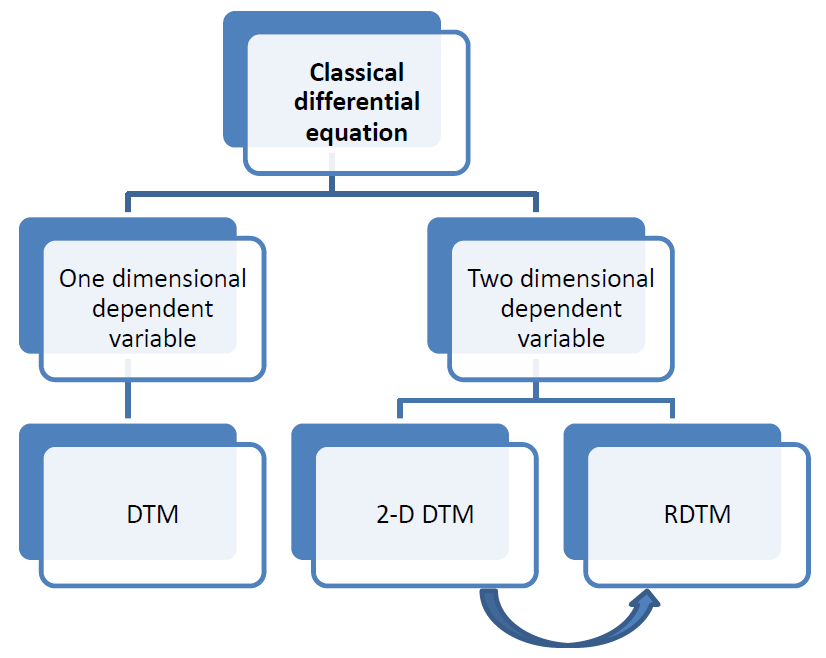

Figure 1. An overview of differential transforms method 
International Journal of Mathematical, Engineering and Management Sciences

Vol. 4, No. 1, 170-181, 2019

https://dx.doi.org/10.33889/IJMEMS.2019.4.1-015

\section{Fractional DTM}

With the dawn of fractional calculus, DTM also got modified to cater the problems involving arbitrary derivatives/integrals. The most captivating part of fractional calculus is its ways to define the fractional derivative. There's no unique definition, unlike traditional derivative.

The fractional integral has its origin in complex analysis (Miller et al., 1993) in the repeated integration of a function $\delta(t)$ due to Cauchy. Formally the fractional integral due to Riemann Liouville (RL) is defined as follows:

Let $\gamma$ be some arbitrary number and its real part be positive. Let $\delta$ represents a piecewise continuous function on domain $(0, \infty)$ and integrable on any finite subinterval of $[0, \infty)$. Then RL fractional integral of $\delta(t)$ of order $q$ is

$J^{\gamma} \delta(t)=D^{-\gamma} \delta(t)=\frac{1}{\Gamma(\gamma)} \int_{0}^{t}(t-\xi)^{\gamma-1} \delta(\xi) d \xi$.

Now knowing the fractional integral which is the fractional antiderivative of the same function, we can proceed to define the fractional derivatives. There are two approaches to calculate $D^{\gamma} \delta(t)$, for instance, $D^{1.5} \delta(t)$ can be obtained from $D^{2} J^{0.5} \delta(t)$ or $J^{0.5} D^{2} \delta(t)$. The first approach gives RL type fractional derivative and the latter gives Caputo type. Formally, the RL fractional derivative (Podlubny, 1999) is given as

$$
\begin{array}{lc}
D^{\gamma} \delta(t)=\frac{d^{m}}{d t^{m}}\left[\frac{1}{\Gamma(m-\gamma)} \int_{0}^{t} \frac{\delta(\xi)}{(t-\xi)^{\gamma-m+1}} d \xi\right], & m-1<\gamma<m \\
D^{\gamma} \delta(t)=\frac{d^{m}}{d t^{m}} \delta(t), & \gamma=m .
\end{array}
$$

And the Caputo's arbitrary derivative is obtained by reversing the order of the derivative and the integral

$$
\begin{array}{lcl}
D^{\gamma} \delta(t)=\frac{1}{\Gamma(m-\gamma)} \int_{0}^{t}(t-\xi)^{m-\gamma-1} \delta^{(m)}(\xi) d \xi, & m-1<\gamma<m \\
D^{\gamma} \delta(t)=\frac{d^{m}}{d t^{m}} \delta(t), & \gamma=m .
\end{array}
$$

For $\gamma=m$ both approaches are equivalent. Unlike the Reimann Liouville derivative, Caputo derivative of a fixed value is zero and the initial constraints retain their integral form. So this approach is comparatively more practical. By this date there are more than twenty other definitions of fractional derivatives, each having one or the other similarity with the traditional derivative operations or improvement over the other. 
International Journal of Mathematical, Engineering and Management Sciences

Vol. 4, No. 1, 170-181, 2019

https://dx.doi.org/10.33889/IJMEMS.2019.4.1-015

Let us assume that $\Phi(r), \Psi(r)$ and $\Omega(r)$ are the fractional differential transforms of $\phi(x), \psi(x)$ and $\omega(x)$ respectively. The results for the considered functions along with their differential transforms (Arikoglu et al., 2007; Arora et al., 2017) are mentioned in Table 4.

Table 4. Fractional differential transforms

\begin{tabular}{|l|l|}
\hline \multicolumn{1}{|c|}{ Original Function } & \multicolumn{1}{|c|}{ Transformed Function } \\
\hline$\phi(x)=\psi(x) \pm \omega(x)$ & $\Phi(r)=\Psi(r) \pm \Omega(r)$ \\
\hline$\phi(x)=c \psi(x)$ & $\Phi(r)=c \Psi(r), c$ is a constant \\
\hline$\phi(x)=\psi(x) \omega(x)$ & $\Phi(r)=\sum_{i=0}^{r} \Psi(i) \Omega(r-i)$ \\
\hline$\phi(x)=D^{\gamma} \psi(x)$ & $\Phi(r)=\frac{\Gamma(\gamma r+\gamma+1)}{\Gamma(\gamma r+1)} \Psi(r+1)$ \\
\hline$\phi(x)=D^{\xi} \psi(x), m-1<\xi \leq m$ & $\Phi(r)=\frac{\Gamma(\gamma r+\xi+1)}{\Gamma(\gamma r+1)} \Psi\left(r+\frac{\xi}{\gamma}\right)$ \\
\hline$\phi(x)=\left(x-x_{0}\right)^{q} ; q=n \gamma ; n \in Z$ & $\Phi(r)=\delta(r-n), \delta$ is Dirac delta function \\
\hline
\end{tabular}

\subsection{2-Dimensional Fractional DTM}

For a function $\phi(x, y)$ represented as a product of two single variable separable functions, in the form, $\phi(x, y)=\delta(x) \theta(y)$, involved in a physical system, defined with the help of fractional derivatives, DTM gets extended. The general differential transform of the function $\phi(x, y)$ in 2dimension (Momani et al., 2008) is given by:

$\Phi_{\gamma, \lambda}(r, s)=\frac{1}{\Gamma(\gamma r+1) \Gamma(\lambda s+1)}\left[\left(D_{x_{0}}^{\gamma}\right)^{r}\left(D_{y_{0}}^{\lambda}\right)^{s} \phi(x, y)\right]_{x_{0}, y_{0}}$.

And then the inverse transform is given as

$$
\phi(x, y)=\sum_{r=0}^{\infty} \Delta_{\gamma}(r)\left(x-x_{0}\right)^{r \gamma} \cdot \sum_{s=0}^{\infty} \Theta_{\lambda}(s)\left(y-y_{0}\right)^{s \lambda}
$$

where $0<\gamma, \lambda \leq 1$ and $\Phi_{\gamma, \lambda},(r, s)=\Delta_{\gamma}(r) . \Theta_{\lambda}(s)$.

The above transformation was precisely called Generalized Differential Transform Method (GDTM) by Odibat and Momani in their letter in 2008 (Odibat et al., 2008). 
International Journal of Mathematical, Engineering and Management Sciences

Vol. 4, No. 1, 170-181, 2019

https://dx.doi.org/10.33889/IJMEMS.2019.4.1-015

Let us assume that $\Phi(r, s), \Psi(r, s)$ and $\Omega(r, s)$ are the fractional differential transforms of $\phi(x, y), \psi(x, y)$ and $\omega(x, y)$ respectively. Table 5 presents the stated functions and their differential transforms.

Table 5. Two-dimensional fractional differential transforms

\begin{tabular}{|l|l|}
\hline \multicolumn{1}{|c|}{ Original Function } & \multicolumn{1}{|c|}{ Transformed Function } \\
\hline$\phi(x, y)=\psi(x, y) \pm \omega(x, y)$ & $\Phi(r, s)=\Psi(r, s) \pm \Omega(r, s)$ \\
\hline$\phi(x, y)=c \psi(x, y)$ & $\Phi(r, s)=c \Psi(r, s), c$ is a constant \\
\hline$\phi(x, y)=\psi(x, y) \omega(x, y)$ & $\Phi(r, s)=\sum_{i=0}^{r} \sum_{j=0}^{s} \Psi(i, s-j) \Omega(r-i, j)$ \\
\hline$\phi(x, y)=D^{\gamma} \psi(x, y)$ & $\Phi(r, s)=\frac{\Gamma(\gamma r+\gamma+1)}{\Gamma(\gamma r+1)} \Psi(r+1, s)$ \\
\hline$\phi(x, y)=\left(x-x_{0}\right)^{m \gamma}\left(y-y_{0}\right)^{n \lambda}$ & $\Phi(r, s)=\delta(r-m) \delta(s-n), \delta$ is Dirac delta function \\
\hline
\end{tabular}

\subsection{Modified fractional DTM}

As there is RDTM for 2-dimensional DTM, there is modified fractional differential transform method (MFDTM) for two dimensional fractional DTM. As the method of differential transform is founded upon the Taylor series for all variables, it leads to tedious calculations. To tackle this problem MFDTM was devised (Aruna et al., 2014) with respect to the independent variables for the function $\phi(x, t)$.

Suppose that the variable of interest is $t$, then the Taylor series of the function $\phi(x, t)$ is defined as:

$$
\phi(x, t)=\sum_{s=0}^{\infty} \frac{1}{\Gamma(\gamma s+1)}\left[\frac{\partial^{\gamma s} \phi(x, t)}{\partial t^{\gamma s}}\right]_{t=t_{0}}\left(t-t_{0}\right)^{\gamma s} .
$$

The differential transform of the function $\phi(x, t)$ is thus given as

$$
\Phi_{\gamma, 1}(x, t)=\sum_{s=0}^{\infty} \frac{1}{\Gamma(\gamma s+1)}\left[\frac{\partial^{\gamma s} \phi(x, t)}{\partial t^{\gamma s}}\right]_{t=t_{0}} .
$$

The modified inverse transform is then given as $\phi(x, t)=\sum_{s=0}^{\infty} \Phi_{\gamma, 1}(x, s)\left(t-t_{0}\right)^{\gamma s}$. The rules for MFDTM are mentioned in Table 6. 
International Journal of Mathematical, Engineering and Management Sciences

Vol. 4, No. 1, 170-181, 2019

https://dx.doi.org/10.33889/IJMEMS.2019.4.1-015

Table 6. Modified fractional differential transforms

\begin{tabular}{|l|l|}
\hline \multicolumn{1}{|c|}{ Original Function } & \multicolumn{1}{|c|}{ Transformed Function } \\
\hline$\phi(x, t)=\psi(x, t) \pm \omega(x, t)$ & $\Phi(r, s)=\Psi(r, s) \pm \Omega(r, s)$ \\
\hline$\phi(x, t)=c \psi(x, t)$ & $\Phi(r, s)=c \Psi(r, s), c$ is a constant \\
\hline$\phi(x, t)=\frac{\partial^{\gamma} \psi(x, t)}{\partial t^{\gamma}}, 0<\gamma \leq 1$ & $\Phi(r, s)=\frac{\Gamma(\gamma s+\gamma+1)}{\Gamma(\gamma s+1)} \Psi(r, s+1)$ \\
\hline$\phi(x, t)=\frac{\partial \psi(x, t)}{\partial x}$ & $\Phi(r, s)=(k+1) \Psi(r+1, s)$ \\
\hline$\phi(x, t)=\left(x-x_{0}\right)^{m}\left(t-t_{0}\right)^{n \gamma}$ & $\Phi(r, s)=\delta(r-m) \delta(s \gamma-n), \delta_{\text {is Dirac delta function }}$ \\
\hline
\end{tabular}



Figure 2. An overview of fractional differential transform method

Figure 1 and Figure 2 give an idea about the basic types of DTM. Having defined the main frameworks for traditional and fractional differential equation, we can take the evolution of the DTM to next level.

\section{Extensions in DTM}

With time, DTM has undergone many problem-specific improvements. Following are some of the major improvements:

In 2004, Ayaz (2004) extended the DTM to tri-dimensional DTM and solved system of linear and non linear pde's. The basic definition of the 3-dimensional differential transform of a function $\omega(x, y, t)$ is 
International Journal of Mathematical, Engineering and Management Sciences

Vol. 4, No. 1, 170-181, 2019

https://dx.doi.org/10.33889/IJMEMS.2019.4.1-015

$\Omega(r, s, u)=\frac{1}{r ! s ! u !}\left(\frac{\partial^{r+s+u} w(x, y, t)}{\partial x^{r} \partial y^{s} \partial t^{u}}\right)_{(0,0,0)}$.

and the inverse differential transform of $\Omega(r, s, u)$ is

$\omega(x, y, t)=\sum_{r=0}^{\infty} \sum_{s=0}^{\infty} \sum_{m=0}^{\infty} \Omega(r, s, u) x^{r} y^{s} t^{u}$.

Table 7 summarizes the rules for 3-dimensional DTM.

Table 7. Three dimensional differential transforms

\begin{tabular}{|l|l|}
\hline \multicolumn{1}{|c|}{ Original Function } & \multicolumn{1}{|c|}{ Transformed Function } \\
\hline$\phi(x, y, t)=\psi(x, y, t) \pm \omega(x, y, t)$ & $\Phi(r, s, u)=\Psi(r, s, u) \pm \Omega(r, s, u)$ \\
\hline$\phi(x, y, t)=c \psi(x, y, t)$ & $\Phi(r, s, u)=c \Psi(r, s, u), c$ is a constant \\
\hline$\phi(x, y, t)=\psi(x, y, t) \omega(x, y, t)$ & $\Phi(r, s, u)=\sum_{i=0}^{r} \sum_{j=0}^{s} \sum_{p=0}^{u} \Psi(i, s-j, u-p) \Omega(r-i, j, p)$ \\
\hline$\phi(x, y, t)=\frac{\partial^{m+n+l} \psi(x, y, t)}{\partial x^{m} \partial y^{n} \partial t^{l}}$ & $\Phi(r, s, u)=\frac{(r+m) !(s+n) !(u+l) !}{r ! s ! u !} \Psi(r+m, s+n, u+l)$ \\
\hline$\phi(x, y, t)=x^{m} y^{n} t^{l}$ & $\Phi(r, s, u)=\delta(r-m) \delta(s-n) \delta(u-l), \delta$ is Dirac delta function \\
\hline
\end{tabular}

In 2005, Arikoglu and Ozkol (2005) developed the DTM for the traditional integro differential equations. In 2010, Erturk and Momani (2010) had developed a conceptual structure to get solutions for fractional integro-differential equations in analytical form. The main results are presented in Table 8. In 2010 only, Nazari and Shahmorad (2010) used FDTM to find the solution of fractional integro-differential equations with non-local boundary constraints.

Table 8. Integro differential transforms

\begin{tabular}{|l|l|}
\hline \multicolumn{1}{|c|}{ Original function } & \multicolumn{1}{|c|}{ Transformed function } \\
\hline$\phi(x)=\int_{x_{0}}^{x} \psi(t) d t$ & $\Phi(r)=\frac{1}{\gamma r} \Psi\left(r-\frac{1}{\gamma}\right)$ \\
\hline$\phi(x)=\psi(x) \int_{x_{0}}^{x} \omega(t) d t$ & $\Phi(r)=\sum_{r_{1}=\frac{1}{\gamma}}^{r} \frac{\Omega\left(r_{1}-\frac{1}{\gamma}\right)}{\gamma r_{1}} \Psi\left(r-r_{1}\right), \quad r \geq \frac{1}{\gamma}$ \\
\hline$\phi(x)=\int_{x_{0}}^{x} \psi_{1}(t) \psi_{2}(t) \ldots \psi_{n}(t) d t$ & $\Phi(r)=\frac{1}{\gamma r} \sum_{r_{n-1}=0}^{r-\frac{1}{\gamma}} \sum_{r_{n-2}=0}^{r_{n-1}} \ldots \sum_{r_{1}=0}^{r_{2}} \Psi_{1}\left(r_{1}\right) \Psi_{2}\left(r_{2}-r_{1}\right) \ldots \Psi_{n-1}\left(r_{n-1}-r_{n-2}\right) \Psi_{n}\left(r-r_{n-1}-\frac{1}{\gamma}\right)$ \\
\hline
\end{tabular}


International Journal of Mathematical, Engineering and Management Sciences

Vol. 4, No. 1, 170-181, 2019

https://dx.doi.org/10.33889/IJMEMS.2019.4.1-015

In 2012, Gökdogan et al. proposed the adaptive MsDTM which is an approach for multi-step differential transformation method (MsDTM). In 2017, Al-Smadi et al. extended this work for partial and fractional differential equations. This multi-step approach is quite simple in obtaining the approximate solutions in forms of a rapidly convergent formula over a long time interval. Multi-step reduced DTM has emerged as a powerful, plain, and promising technique in finding the analytic approximate solution for a wider family of fractional PDEs.

For pure mathematicians, a series solution is useless unless its convergence is established. This issue was resolved by Odibat et al. beautifully in 2016, wherein they presented a trustworthy approach for discussing the convergence of method and also derived a sufficient condition for convergence with application to fractional differential equations having non-linear terms.

\subsection{Applications}

Though this method has been used in its various forms to solve a number of linear and non-linear traditional and fractional differential equations, some of them are mentioned below:

In 2011, Ibis et al. (2011) used FDTM to solve the differential algebraic equations of fractional order. They also made comparisons between the homotopy analysis method (HAM), fractional differential transform method (FDTM) and the exact solutions. In 2011, Soltanalizadeh (2011) solved 1-dimensional telegraph equation using DTM. Benhammouda et al. (2014) modified RDTM for partial differential algebraic equations. Abazari et al. (2014) extended 2-dimensional DTM and applied on proportional delay infused partial differential equations. In the same year, Momani et al. used a slight modification of GDTM (Momani et al., 2014) to solve the fractionalorder multiple chaotic Fitz Hugh-Nagumo (FHN) neurons model. This method gave an analytical form of the solution within each time interval which was not possible using techniques like the fourth-order Runge-Kutta method. In 2015, Bansal et al. obtained approximate solutions of the fractional order Riccati differential equation. In 2016, Abuteen et al. (2016) worked upon fractional Klein-Gordon equations using fractional reduced DTM.

Recently, some researchers have experimented with DTM by adopting other than Caputo's definition. In the year 2016, DTM was explained with a promising definition of composite derivative (Garg et al., 2016) and it was used in solving fractional Fokker - Planck Equation. In 2017, similar work was done in DTM using conformable (Unal et al., 2017) fractional derivative.

\section{Conclusion}

Due to much utility of fractional calculus in mechanical engineering (Hatami et al., 2017), chemical engineering, biological engineering, magneto hydrodynamics etc, the need of the hour is a method that is effectively capable to solve linear and non-linear differential equations of arbitrary order. The differential transform method had been an efficient method and has evolved a lot from Zhou's conceptualization to the multi-step reduced DTM. It has been modified in various forms to solve the differential equations: linear and non-linear; one variable and multi-variables; ordinary, partial and fractional.

The contemporary authors are now trying different definitions of fractional derivatives. The quest of a perfect definition of fractional derivative is unending, and it keeps on the curiosity to work upon DTM with various definitions for better solutions. This work is an attempt to summarize the progress in DTM in last few years. 
International Journal of Mathematical, Engineering and Management Sciences

Vol. 4, No. 1, 170-181, 2019

https://dx.doi.org/10.33889/IJMEMS.2019.4.1-015

\section{Conflict of Interest}

The author(s) confirm that this article contents have no conflict of interest.

\section{Acknowledgement}

We are grateful to all the reviewers for their encouraging comments and valuable corrections.

\section{References}

Abazari, R., \& Ganji, M. (2011). Extended two-dimensional DTM and its application on nonlinear PDEs with proportional delay. International Journal of Computer Mathematics, 88(8), 1749-1762.

Abuteen, E., Freihat, A., Al-Smadi, M., Khalil, H., \& Khan, R. A. (2016). Approximate series solution of nonlinear, fractional Klein Gordon equations using fractional reduced differential transform method. Journal of Mathematics and Statistics, 12(1), 23-33.

Al-Smadi, M., Freihat A., Khalil H., Momani, S., \& Khan R. A. (2017). Numerical multistep approach for solving fractional partial differential equations. International Journal of Computational Methods, 14(3), 1750029 (15 pages).

Arikoglu, A., \& Ozkol, I. (2005). Solution of boundary value problems for integro-differential equations by using differential transform method. Applied Mathematics and Computation, 168(2), 1145-1158.

Arikoglu, A., \& Ozkol, I. (2007). Solution of fractional differential equations by using differential transform method. Chaos, Solitons \& Fractals, 34(5), 1473-1481.

Arora, G., \& Pratiksha. (2017). Solution of the Bagley Torvik equation by fractional DTM. In AIP Conference Proceedings, $1860(1), 020032$.

Aruna, K., \& Kanth, A. R. (2014). Two-dimensional differential transform method and modified differential transform method for solving nonlinear fractional Klein-Gordon equation. National Academy Science Letters, 37(2), 163-171.

Ayaz, F. (2003). On the two-dimensional differential transform method. Applied Mathematics and Computation, 143(2-3), 361-374.

Ayaz, F. (2004). Solutions of the system of differential equations by differential transform method. Applied Mathematics and Computation, 147(2), 547-567.

Bansal, M. K., \& Jain, R. (2015). Application of generalized differential transform method to fractional order riccati differential equation and numerical result. International Journal of Pure and Applied Mathematics, 99(3), 355-366.

Benhammouda, B., Vazquez-Leal, H., \& Sarmiento-Reyes, A. (2014). Modified reduced differential transform method for partial differential-algebraic equations. Journal of Applied Mathematics, 2014,9 pages.

Biazar, J., \& Eslami, M. (2010). Differential transform method for quadratic Riccati differential equation. International Journal of Nonlinear Science, 9(4), 444-447.

Cetinkaya, A., Kıymaz, O., \& Caml,, J. (2011). Solutions of nonlinear PDE's of fractional order with generalized differential transform method. International Mathematical Forum, 6(1), 39-47.

Chen, C. O. K., \& Ho, S. H. (1999). Solving partial differential equations by two-dimensional differential transform method. Applied Mathematics and Computation, 106(2-3), 171-179.

Erturk, V. S. (2007). Application of differential transformation method to linear sixth-order boundary value problems. Applied Mathematical Sciences, 1(2), 51-58.

Erturk, V. S., \& Momani, S. (2008). Solving systems of fractional differential equations using differential transform method. Journal of Computational and Applied Mathematics, 215, 142-151. 
International Journal of Mathematical, Engineering and Management Sciences

Vol. 4, No. 1, 170-181, 2019

https://dx.doi.org/10.33889/IJMEMS.2019.4.1-015

Erturk, V. S., \& Momani, S. (2010). On the generalized differential transform method: Application to fractional integro-differential equations. Studies in Nonlinear Sciences, 1(4), 118-126.

Erturk, V. S., Momani S., \& Odibat Z. (2008). Application of generalized differential transform method to multi-order fractional differential equations. Communications in Nonlinear Science and Numerical Simulation, 13, 1642-1654.

Garg, M., \& Manohar, P. (2016). Generalisation of Taylor's formula and differential transform method for composite fractional derivative. Indian Journal of Industrial and Applied Mathematics, 7(1), 65-75.

Gokdogan A., Merdana M., \& Yildirim, A. (2012). Adaptive multi-step differential transformation method to solving nonlinear differential equations. Mathematical and Computer Modelling, 55, 761-769.

Hatami, M., Ganji, D. D., \& Sheikholeslami, M. (2017). Differential transformation method for mechanical engineering problems. Academic Press. ISBN: 9780128053409.

Ibis, B., Bayram M., \& Agargn, A. G. (2011). Applications of fractional differential transform method to fractional differential-algebraic equations. European Journal of Pure and Applied Mathematics, 4(2), 129-141.

Keskin, Y., \& Oturanc, G. (2009). Reduced differential transform method for partial differential equations. International Journal of Nonlinear Sciences and Numerical Simulation, 10(6), 741-749.

Miller, K. S., \& Ross, B. (1993). An introduction to the fractional calculus and fractional differential equations. Wiley-Interscience.

Mirzaee, F. (2011). Differential transform method for solving linear and non-linear systems of ordinary differential equations. Applied Mathematical Sciences, 5(70), 3465-3472.

Momani, S., \& Odibat, Z. (2008). A novel method for nonlinear fractional partial differential equations: Combination of DTM and generalized Taylor's formula. Journal of Computational and Applied Mathematics, 220, 85-95.

Momani, S., Freihat A., \& AL-Smadi, M. (2014). Analytical study of fractional-order multiple chaotic FitzHugh-Nagumo neurons model using multistep generalized differential transform method. Abstract and Applied Analysis, 2014, Article ID 276279.

Nazari, D., \& Shahmorad, S. (2010). Application of the fractional differential transform method to fractional-order integro-differential equations with nonlocal boundary conditions. Journal of Computational and Applied Mathematics, 234, 883-891.

Odibat, Z. M., Kumar, S., Shawagfeha, N., Alsaedi, A., \& Hayat, T. (2016). A study on the convergence conditions of generalized differential transform method. Mathematical Methods in Applied Sciences, $40,40-48$.

Odibat, Z., \& Momani, S. (2008). A generalized differential transform method for linear partial differential equations of fractional order. Applied Mathematics Letters, 21, 194-199.

Podlubny, I. (1999). Fractional differential equations. Academic Press.

Soltanalizadeh, B. (2011). Differential transformation method for solving one space- dimensional telegraph equation. Computational and Applied Mathematics, 30(3), 639-653.

Unal, E., \& Gokdogan, A. (2017). Solution of conformable fractional ordinary differential equations via differential transform method. Optik - International Journal for Light and Electron Optics, 128, 264273.

Zhou, J. K. (1986). Differential transformation and its application for electrical circuits (in Chinese). Huazhong University Press. 